\title{
Response of Baroclinic Life Cycles to Barotropic Shear
}

\author{
Dennis L. Hartmann And Peter Zuercher \\ Department of Atmospheric Sciences, University of Washington, Seattle, Washington
}

(Manuscript received 29 July 1996, in final form 25 February 1997)

\section{ABSTRACT}

\begin{abstract}
Cyclonic barotropic shear of incrementally increasing magnitude is imposed on an idealized midlatitude jet, and the life cycles of baroclinically unstable wavenumber 6 perturbations growing on these jets are studied. When the barotropic shear parameter passes a critical value, the life cycle makes an abrupt transition from anticyclonic to cyclonic behavior. The abrupt transition in behavior is most evident in the barotropic decay of eddy kinetic energy, in the structure of the eddies as seen in potential vorticity maps, and in the nature of the zonal flow accelerations produced during the life cycle. It is suggested that the abrupt transition can be interpreted as arising from a positive feedback between eddy propagation and eddy-induced zonal flow accelerations. Dependences on the zonal scale of the eddy are also investigated. Wavenumber 8 exhibits cyclonic behavior, while wavenumber 4 exhibits anticyclonic behavior for all values of cyclonic shear considered. This dependence on zonal scale is consistent with predictions of linear WKB wave propagation theory. The sharpness of the transition in life cycle behavior has implications for medium-range predictability and for the generation of lowfrequency variability in the atmosphere.
\end{abstract}

\section{Introduction}

Simmons and Hoskins (1980, hereafter SH) made a pioneering study of the effect of barotropic meridional shear on baroclinic wave life cycles in idealized numerical experiments. In particular they noted what they called an "anomalous case" in which the addition of cyclonic barotropic shear caused little change in the maximum level of eddy energy attained during the life cycle, but caused the kinetic energy to be maintained for a much longer time. Later Thorncroft et al. (1993, hereafter THM) repeated this calculation with a much higher resolution model and performed a more detailed analysis of the nature of the differences between their $\mathrm{LC} 1$ and LC2 cases, which we will refer to here as the anticyclonic and cyclonic cases, respectively. These diagnostics showed that in the anticyclonic case the wave propagates equatorward and is absorbed in the subtropics, whereas in the cyclonic case the wave does not propagate as effectively equatorward, but is trapped in a nonabsorbing waveguide on the cyclonic side of the jet. Using a conservation theorem of Haynes (1988), THM argue that the cyclonic case is an example of nonlinear Rossby wave critical layer reflection. Hartmann (1995), on the basis of numerical simulations by Yu and Hartmann (1993) and an analysis of observa-

Corresponding author address: Dr. Dennis L. Hartmann, Department of Atmospheric Sciences, Box 351640, University of Washington, Seattle, WA 98195.

E-mail: dennis@atmos.washington.edu tions, drew a connection between these two types of wave life cycle behavior and the dominant mode of lowfrequency variability in the Southern Hemisphere, which is an almost barotropic dipolar mode with centers of amplitude of opposite sign at about $40^{\circ} \mathrm{S}$ and $60^{\circ} \mathrm{S}$. The changes in wave life cycle behavior induce changes in zonal flow acceleration that reinforce the original anomaly in barotropic zonal flow. The interaction between the wave life cycle and barotropic shear creates a positive feedback that gives rise to low-frequency variability of the zonal mean flow. An alternative but related theory for low-frequency variability of zonal flow has been given by James and Dodd (1996).

In THM only cyclonic shear parameters of 0 and 10 $\mathrm{m} \mathrm{s}^{-1}$ were considered. In this study we analyze a sequence of experiments in which the shear parameter is increased in small increments from 0 to $10 \mathrm{~m} \mathrm{~s}^{-1}$. We show that the transition from anticyclonic to cyclonic behavior of wave life cycles is very abrupt, occurring across a very narrow range of shear parameter. One interpretation of this abrupt transition is that it arises from a positive feedback between zonal flow accelerations and wave propagation and absorption. This strong positive feedback is consistent with the idea that active feedback between baroclinic wave life cycles and barotropic shear is the critical mechanism for generating low-frequency variability of the type characterized by the dominant mode of low-frequency variability in the Southern Hemisphere extratropics. This feedback gives rise to a kind of intransitive behavior (Lorenz 1964) in which a wide range of zonal mean flows are viable 

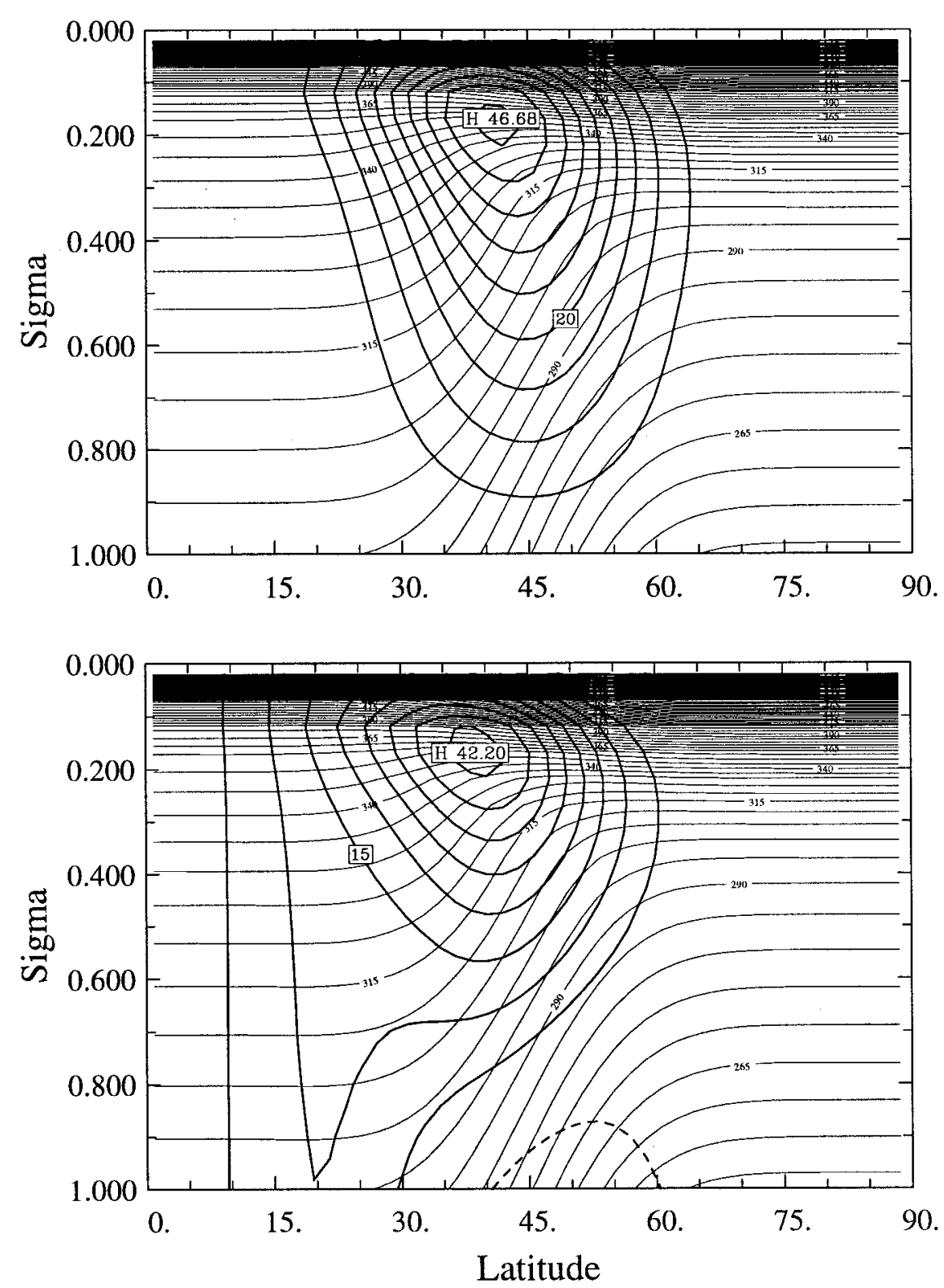

FIG. 1. Meridional cross sections of zonal wind (heavy contours) and potential temperature for the (a) basic $45^{\circ}$ jet and (b) basic jet plus an added barotropic shear with $u_{0}=10 \mathrm{~m} \mathrm{~s}^{-1}$. Contour intervals are $5 \mathrm{~m} \mathrm{~s}^{-1}$ and $5 \mathrm{~K}$.

quasi-steady states, although the stability of these states in the presence of realistic noise levels is uncertain. The sequence of experiments also enables us to better understand the nature of this positive feedback and the transition from anticyclonic to cyclonic behavior.

\section{Model and procedure}

The procedure we will follow is to perform a series of 20-day nonlinear life-cycle experiments starting with a basic $45^{\circ}$ lat baroclinic jet and adding barotropic shear with a specified shape, but with varying magnitude. The baroclinic jet is modeled after SH and THM, including the slight equatorward tilt of the jet with altitude to produce a realistically sloping tropopause. We did a sequence of experiments for two slightly different baroclinic jets. The first jet we constructed tilted equatorward slightly more rapidly and produced an internal reversal of the potential vorticity gradient slightly under and equatorward of the jet maximum. The second jet was modified so that there was no potential vorticity gradient reversal in this region, which made calculations of the index of refraction much cleaner. Both jets showed an abrupt transition from anticyclonic to cyclonic behavior, albeit at different values of the shear parameter. In this paper, we will describe only the results 


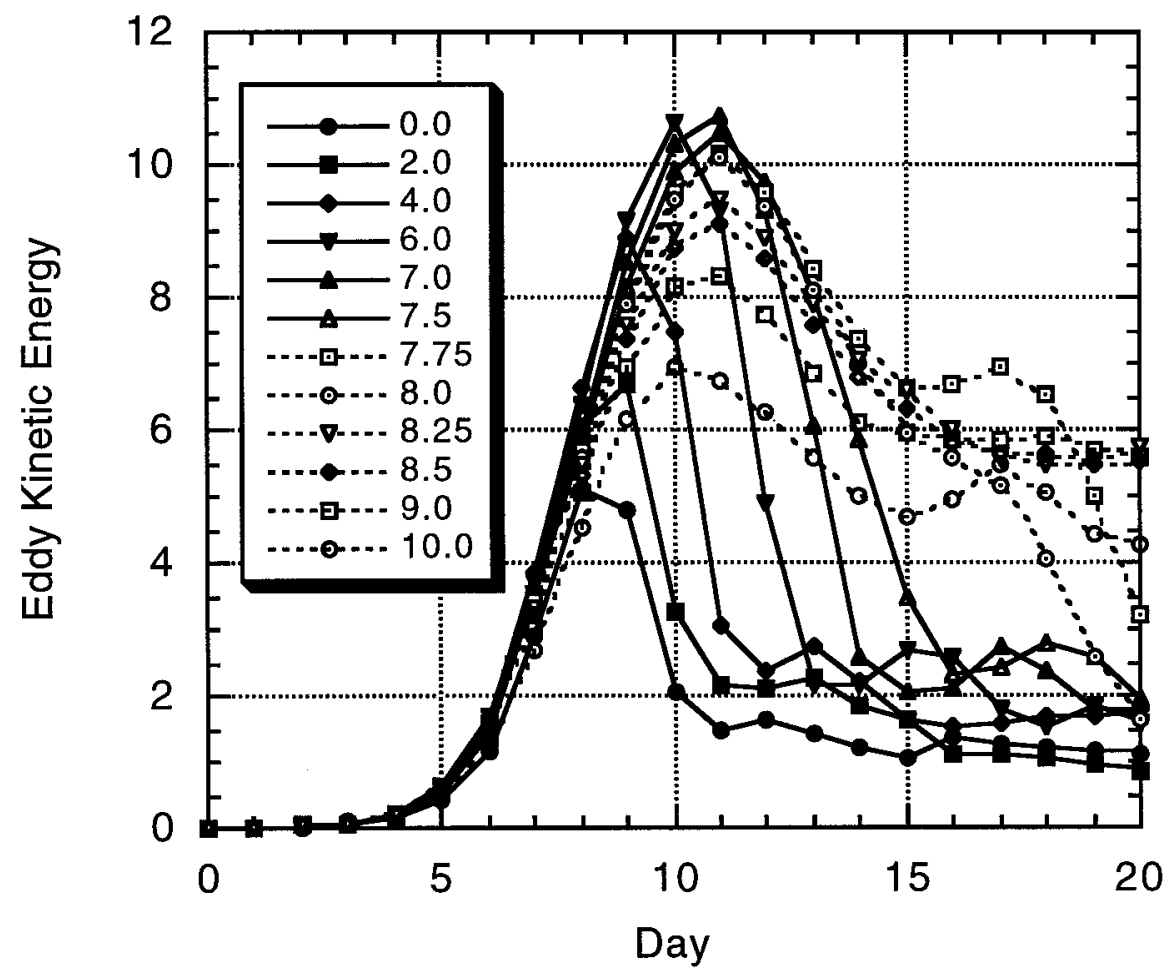

FIG. 2. Hemispheric eddy kinetic energy as a function of time for various values of the shear parameter $u_{0}$ between 0 and $10 \mathrm{~m} \mathrm{~s}^{-1}$. Units are joules $\times 10^{20}$.

for the second baroclinic jet, without the potential vorticity gradient reversal. The results for the basic jet that we will discuss here show less growth and a less sharp transition than the results we will not show, but the basic behavior is very similar, suggesting that our main conclusions are not highly sensitive to details of how the jet is constructed or to the presence or absence of internal potential vorticity gradient reversals. Magnusdottir and Haynes (1996) also found that the baroclinic life-cyle behavior regimes were not dependent on the presence or absence of interior potential vorticity gradient reversals.

The barotropic shear added to the basic $45^{\circ}$ baroclinic jet has a westerly jet at $20^{\circ}$ and an easterly jet at $50^{\circ}$ specified according to the following formula:

$$
\begin{aligned}
& u_{b}(\varphi) \\
& =u_{0}\left\{\exp \left(-\left(\frac{\varphi-20}{12.5}\right)^{2}\right)-\exp \left(-\left(\frac{\varphi-50}{12.5}\right)^{2}\right)\right\} .
\end{aligned}
$$

Here, $\varphi$ is latitude given in degrees, and $u_{0}$ is the shear parameter, which will be varied between 0 and 10. Separate integrations are done for values of $u_{0}$ at 1 or $2 \mathrm{~m}$ $\mathrm{s}^{-1}$ intervals, and then a subset at finer spacing is done to define the sharpness of the transition from anticyclonic to cyclonic behavior. The initialized wind and potential temperature distributions for shear parameters of 0 and $10 \mathrm{~m} \mathrm{~s}^{-1}$ are shown in Fig. 1. A small wavenumber 6 vorticity perturbation is added to the zonal fields to create initial conditions for life-cycle experiments.

The model used to conduct these experiments is a primitive equation, sigma-coordinate model as described in Yu and Hartmann (1993) with T63 spherical harmonic spectral representation of horizontal fields and linear finite element representation of vertical variations. The 15 sigma-coordinate levels used in these experiments are $0.02, .07, .12, .17, .22, .27, .32, .37, .43, .51$, $.6, .7, .8, .9$, and 1.0. Tests show that the results are not especially sensitive to the number or placement of the sigma levels. A significant subset of the experiments have also been done at T42 resolution. Life-cycle behavior shows more rapid growth and sharper transitions for T63 than for T42 model resolutions so that we expect higher resolution than T63 would produce slightly sharper transitions, but no qualitative changes in our conclusions. The only physical process included in the model is a "del-sixth" diffusion with a coefficient of $1.416 \times 10^{26} \mathrm{~m}^{6} \mathrm{~s}^{-1}$, which gives an $e$-folding time of 2 hours at total wavenumber 63 . The time step is 20 min and each integration is continued for 20 days.

\section{Sharpness of the transition to cyclonic behavior \\ a. Eddy kinetic energy and energy conversions}

As a diagnostic of life-cycle behavior, we show the time history of eddy kinetic energy (EKE) in Fig. 2 for 


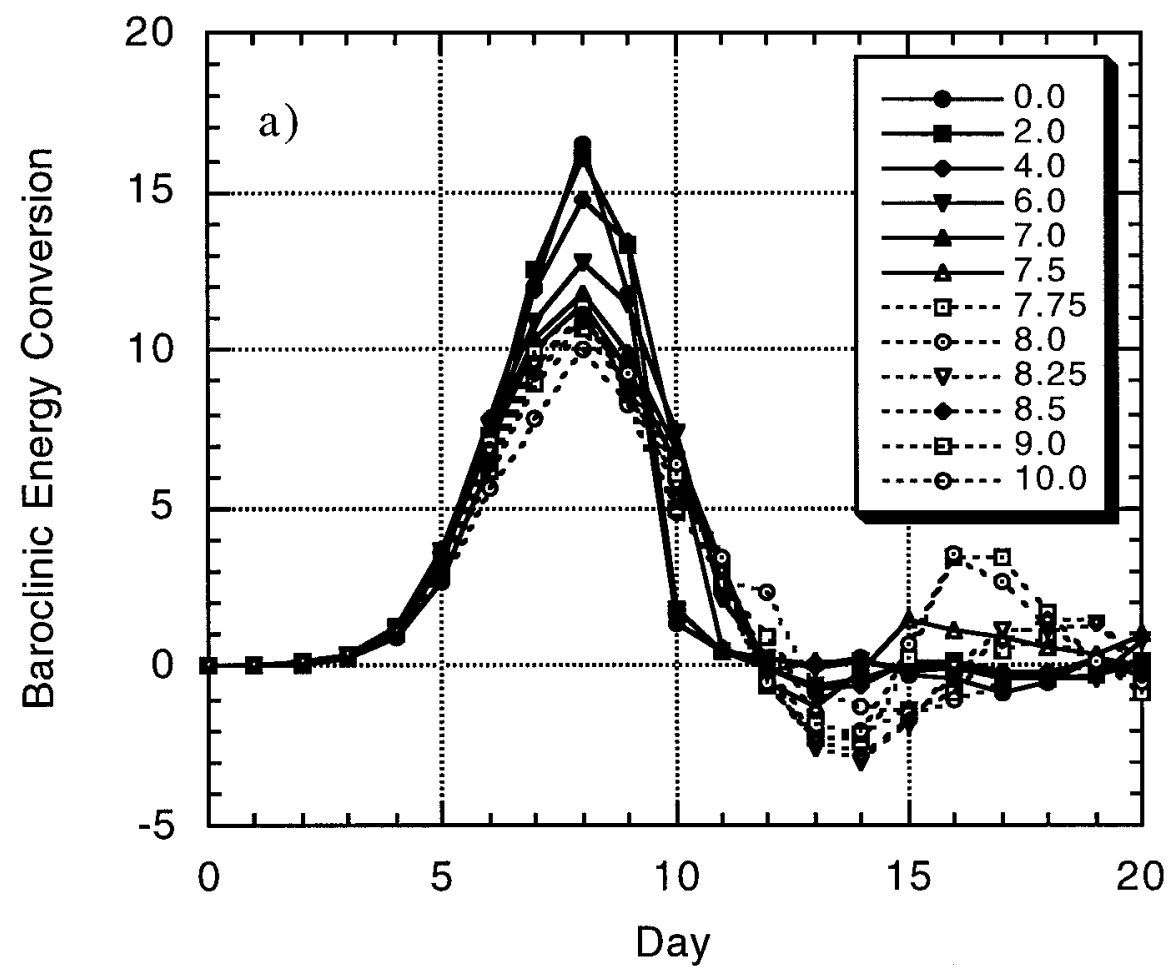

FIG. 3. Energy conversion terms integrated over mass and plotted as a function of time for various values of the shear parameter: (a) baroclinic energy conversion $C_{E}$, (b) barotropic energy conversion $C_{K}$, and (c) total eddy energy conversion $C_{E}+C_{K}$. Units are watts per square meter.

various values of the shear parameter $u_{0}$. The period of exponential growth extends from around day 4 to day 7, followed by leveling off or collapse of the EKE. The peak EKE attained varies between about 5 for $u_{0}=0.0$ and about 11 for $u_{0}=7.0$. At day 16 the eddy kinetic energy falls into two clusters, one near 2 and one near 6 . The transition between these two clusters is rather sharp and falls between $u_{0}=7.5$ and $u_{0}=7.75 \mathrm{~m} \mathrm{~s}^{-1}$. The shear parameters of $u_{0}=7.75$ and $u_{0}=8.0 \mathrm{~m} \mathrm{~s}^{-1}$ yield transition cases that initially show sustained eddy kinetic energy but then fall off after day 16 . These cases show the sensitivity of the behavior of the life cycle for shear parameters near the transition value. The other basic baroclinic jet we studied showed a sharp transition from anticyclonic to cyclonic behavior between $u_{0}=$ 5.25 and $u_{0}=5.5$, and we did not investigate the sensitivity to shear parameter changes smaller than 0.25 (Zuercher 1996). It can be seen that the peak EKE achieved initially increases with the addition of cyclonic barotropic shear from $u_{0}=0$ to about $u_{0}=7$, but then begins to decline with increasing shear. The time of the peak EKE also is increased from day 8 for $u_{0}=0$ to day 11 for $u_{0}=7.0$.

To understand the temporal evolution of eddy kinetic energy in these cases it is useful to compute the massintegrated kinetic energy conversion terms as a function of time. The baroclinic EKE conversion term is given by

$$
C_{E}=-\int \overline{\omega^{\prime} \alpha^{\prime}} d m
$$

and the barotropic EKE conversion term is calculated from

$$
C_{K}=-\int\left\{\frac{\overline{u^{\prime} v^{\prime}}}{a} \frac{\partial}{\partial \varphi}\left(\frac{\bar{u}}{\cos \varphi}\right)+\overline{u^{\prime} \omega^{\prime}} \frac{\partial}{\partial p}\left(\frac{\bar{u}}{\cos \varphi}\right)\right\} d m .
$$

Here $\varphi$ is latitude, $d m$ indicates an integral over the mass of the hemisphere, and the other symbols have their usual meteorological meaning (Lorenz 1967). The response of the kinetic energy and the conversion terms to cyclonic shear is in general accord with the results shown by SH. Cyclonic shear has a weak, but negative, impact on baroclinic conversion (Fig. 3a), but as the shear is increased the maximum EKE achieved increases because the barotropic decay event that collapses the EKE is delayed (Figs. 3b and 3c). When the initial cyclonic shear is larger than a threshold value, the barotropic decay event fails to occur entirely and the EKE remains high for an extended period. Despite the absence of a strong barotropic decay event, the peak EKE achieved declines with increasing shear in the cyclonic behavior regime because the baroclinic growth weakens significantly, and a weak baroclinic decay event devel- 

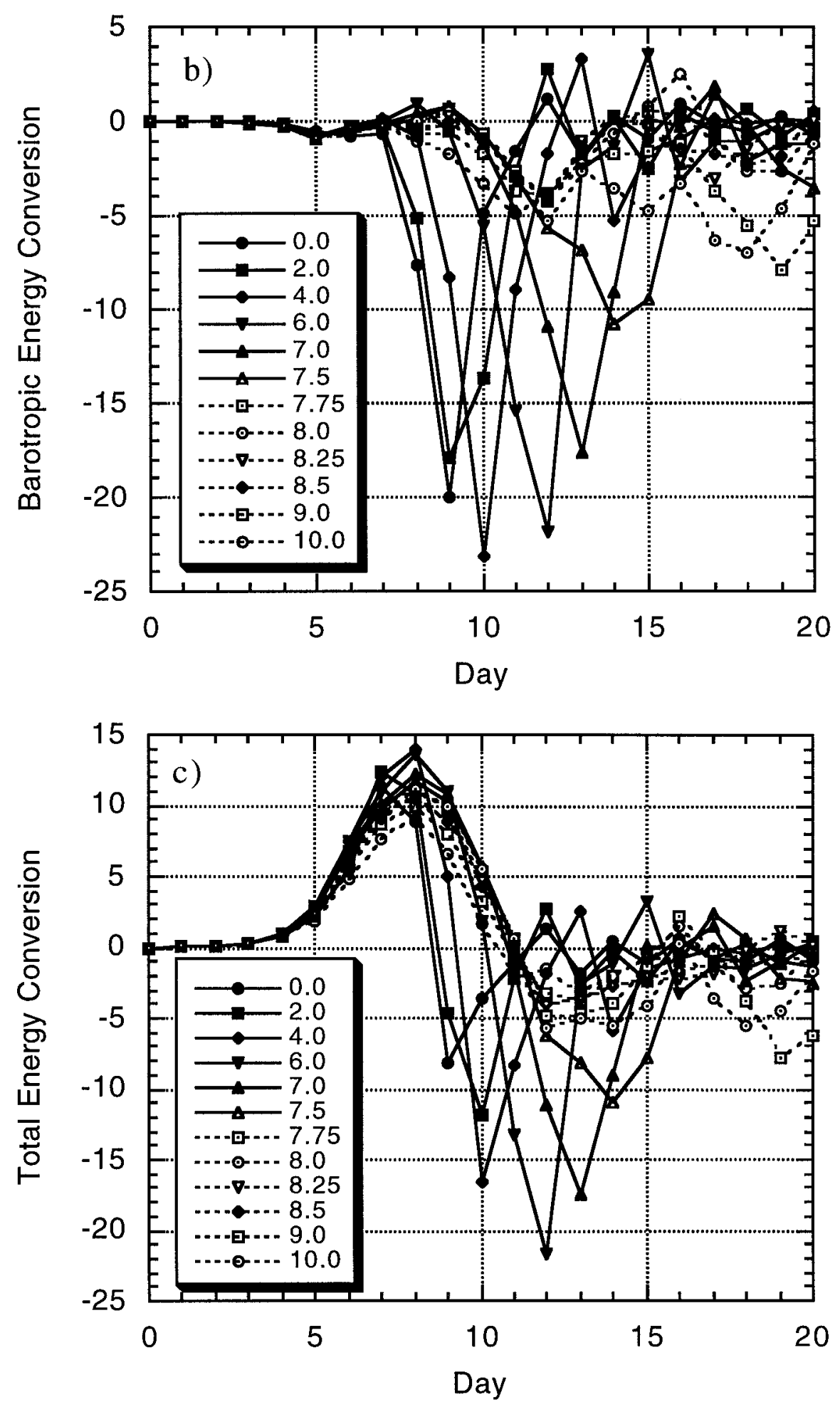

FIG. 3 (Continued)

ops around day 13. James (1987) has discussed the dependence of baroclinic growth rate on barotropic shear. To further emphasize the sharpness of the transition from anticyclonic to cyclonic behavior we show in Fig.
4 the eddy kinetic energy averaged over days 16-20 and the barotropic energy conversion integrated over days 1-20, each plotted as functions of shear parameter. Both variables show a sharp change between shear parameters 


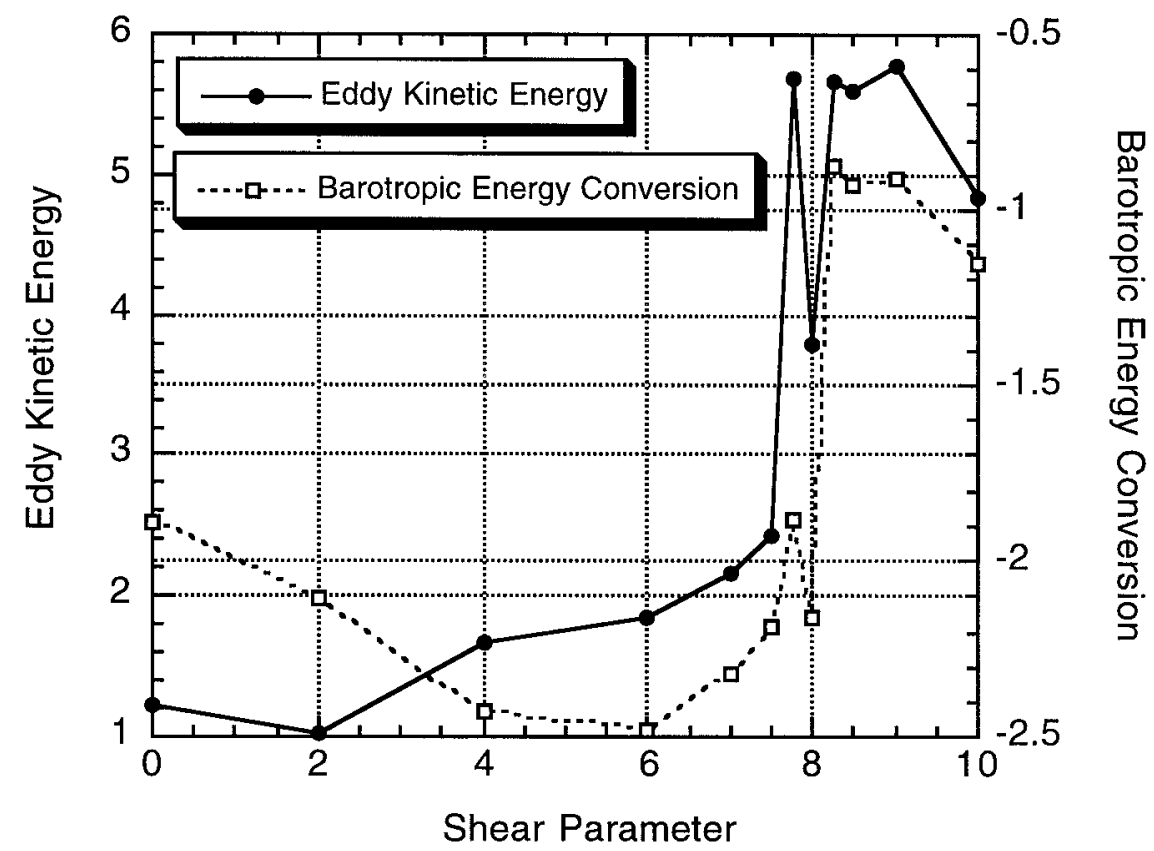

FIG. 4. (a) Mass-integrated eddy kinetic energy averaged over days 16-20 and (b) mass-integrated barotropic energy conversion averaged over days 1-20 plotted as functions of shear parameter $u_{0}$. Units are joules $\times 10^{20}$ and watts per square meter, respectively.

of 7.5 and 8.25 , with somewhat inconsistent behavior for parameters 7.75 and 8.0 , because for these cases the level of EKE and the barotropic conversion terms were still changing near the end of the 20-day experiment.

\section{b. Potential vorticity maps and wave structure}

The eddy kinetic energy shows a sharp transition from anticyclonic to cyclonic behavior as the shear parameter is increased from 7.5 to $8.25 \mathrm{~m} \mathrm{~s}^{-1}$, and this transition is also very obvious in the flow structure revealed by potential vorticity (PV) maps (Hoskins et al. 1985). The $\mathrm{PV}$ evolution differences we see between the 7.5 to 8.25 $\mathrm{m} \mathrm{s}^{-1}$ cases are very similar to the differences between the 0 and $10 \mathrm{~m} \mathrm{~s}^{-1}$ shear parameter cases described by THM. Cases for shear parameters between 0 and $7.5 \mathrm{~m}$ $\mathrm{s}^{-1}$ show some continuous changes, such as the upstream tilt of the trough in the early part of the simulation, which is greater in the $0 \mathrm{~m} \mathrm{~s}^{-1}$ than the $7.5 \mathrm{~m} \mathrm{~s}^{-1}$ shear parameter case (not shown). The nonlinear evolution to the ending state changes abruptly at a critical value of the shear parameter, however.

Figure 5 shows the PV evolution at $335 \mathrm{~K}$ for the case of $u_{0}=7.5 \mathrm{~m} \mathrm{~s}^{-1}$ and Fig. 6 shows the result for $u_{0}=8.25 \mathrm{~m} \mathrm{~s}^{-1}$. A very small change of $0.75 \mathrm{~m} \mathrm{~s}^{-1}$ in the barotropic component of the original zonal wind field gives a radically different flow evolution during the later stages of the life cycle. At day 7 the two cases are very difficult to distinguish. By day 11 a small difference is becoming evident, with the $7.5 \mathrm{~m} \mathrm{~s}^{-1}$ case showing a slightly more elongated trough and slightly more upstream tilt (SW-NE), particularly at its tropical extremity. After day 11 the 7.5 (anticyclonic) case shows rapid thinning of the trough and enhanced upstream tilt of the trough, so that by day 15 a substantial portion of the trough is being sheared off in a wave breaking event on the equatorward side of the jet. In contrast, the $8.25 \mathrm{~m} \mathrm{~s}^{-1}$ shear parameter case never shows much upstream tilt and eventually develops rounded, closed cyclones on the cyclonic side of an undular jet and its associated strong PV gradient.

\section{Mechanism of the transition}

\section{a. Index of refraction}

Linear theory gives some insight into the continuous response to the shear parameter for the early part of the simulation up to about day 7. As a diagnostic of linear wave propagation, THM used the modified refractive index $K$, given by

$$
K=\frac{a^{2} \bar{q}_{y}}{\bar{U}-c}
$$

where $K$ is related to the true index of refraction by

$$
l^{2}=K^{2}-m^{2},
$$

$m$ is the zonal wavenumber, $l$ is the meridional wavenumber, $\bar{q}_{y}$ is the quasigeostrophic potential vorticity gradient, and $c$ is the phase speed of the wave (e.g., see Matsuno 1970; Hoskins and Karoly 1981; Karoly and Hoskins 1982). Although the waves have a similar me- 

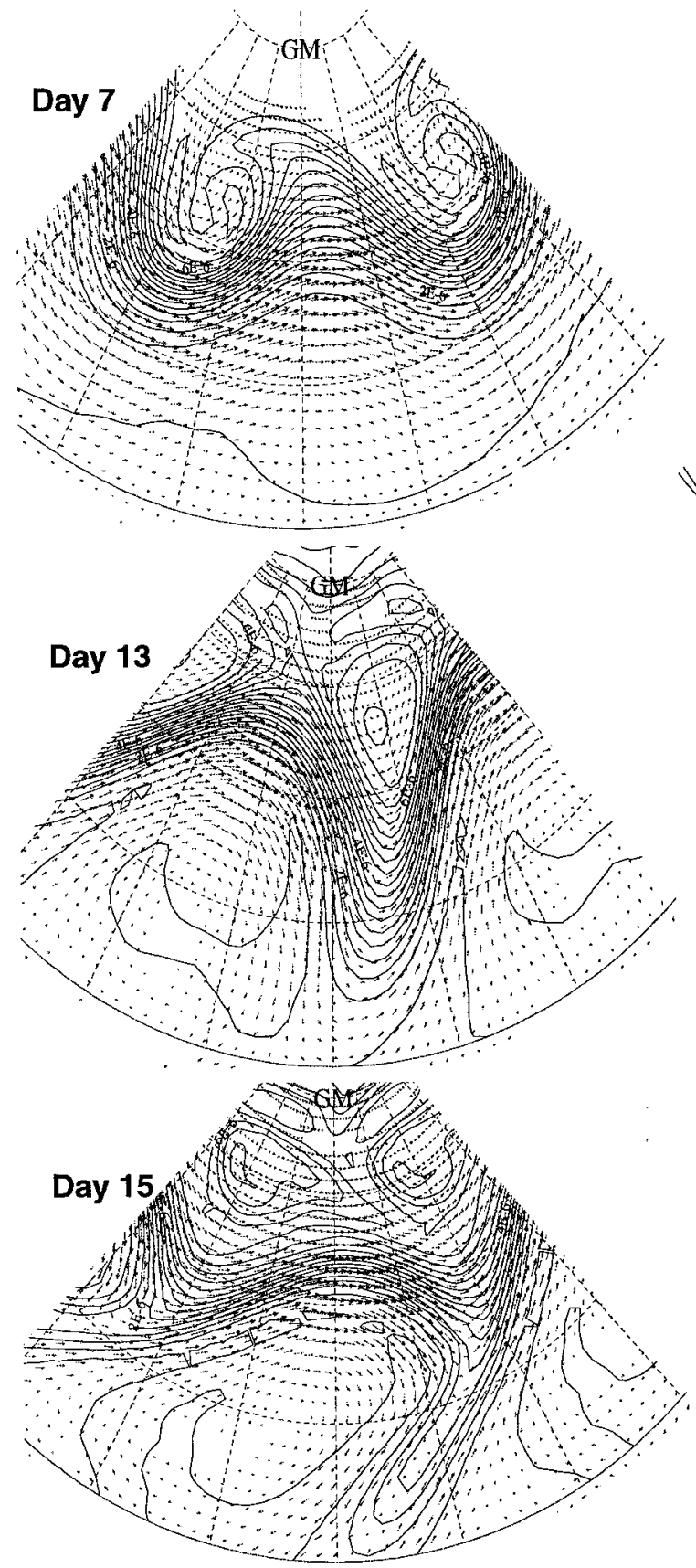

\section{$7.5 \mathrm{~m} / \mathrm{s}$ shear}
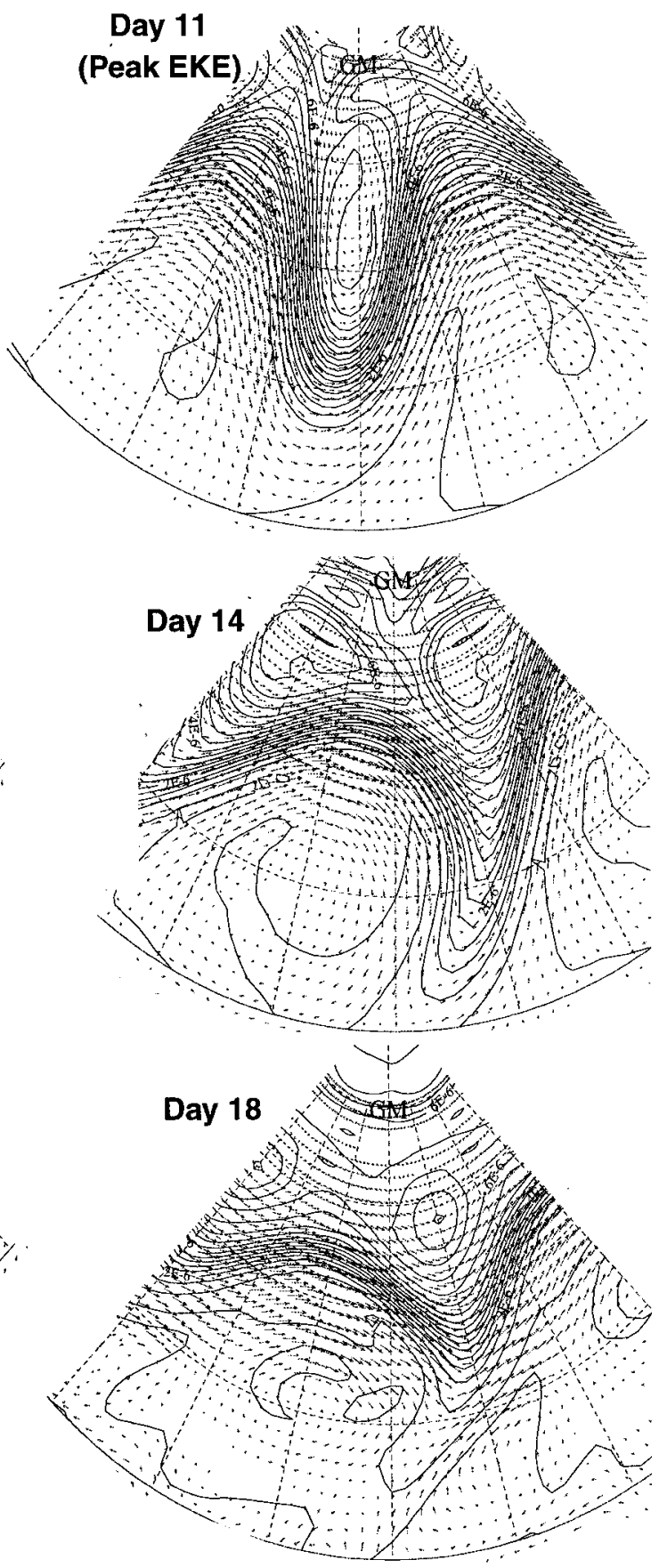

FIG. 5. Contours of PV and velocity vectors on the 335-K isentropic surface for selected days of the $u_{0}=7.5 \mathrm{~m} \mathrm{~s}^{-1}$ case. The PV contour interval is $5.0 \times 10^{-7} \mathrm{~K} \mathrm{~kg}^{-1} \mathrm{~m}^{2} \mathrm{~s}^{-1}$. The bottom latitude circle is $15^{\circ} \mathrm{N}$ and latitude-longitude lines are drawn every $15^{\circ}$.

ridional scale to the zonal wind variations so that the scaling arguments for WKB theory do not strictly apply, we investigate what predictions that theory makes and ask whether these predictions agree with the behavior of the nonlinear model. According to the linear WKB theory, Rossby waves will be refracted toward the gradient of the index of refraction and so from smaller to larger values of $K$. Ray theory predicts that the wave 

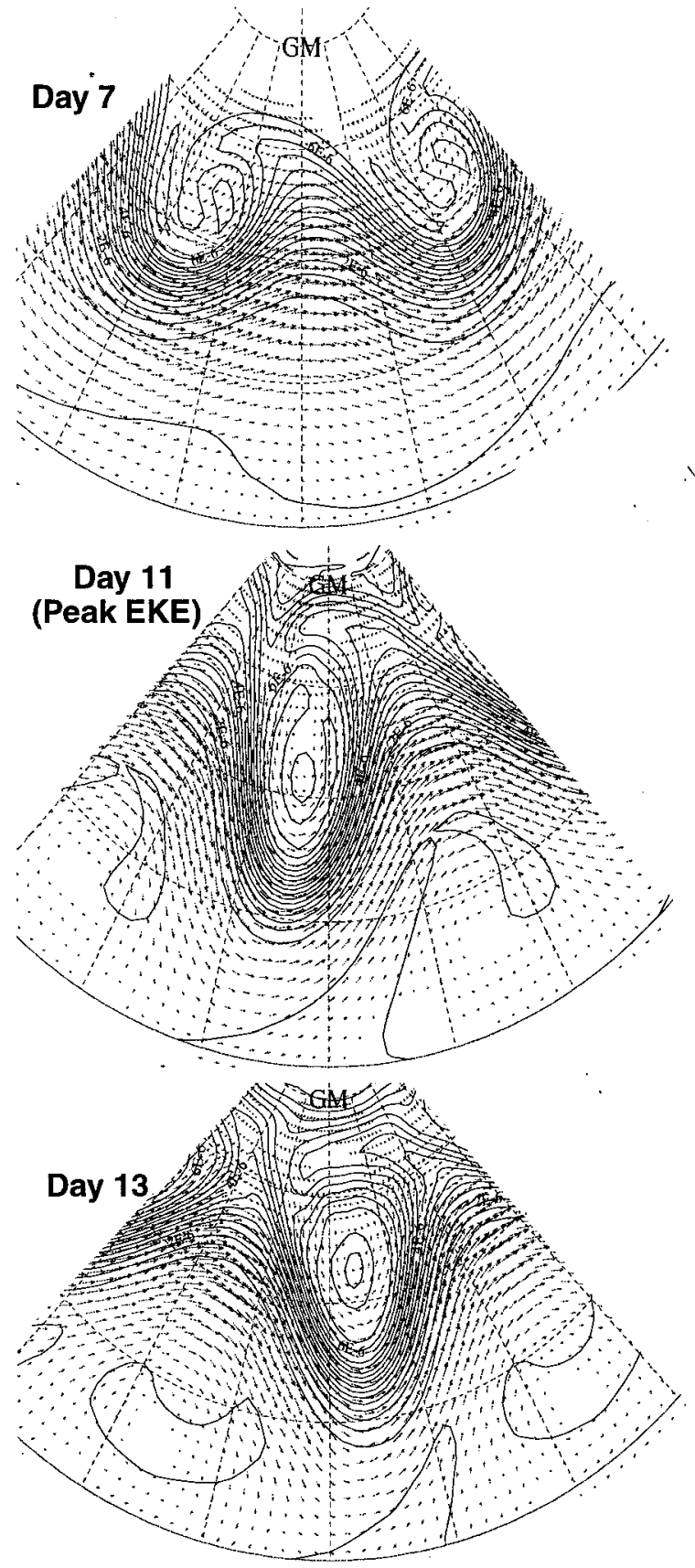

\section{$8.25 \mathrm{~m} / \mathrm{s}$ shear}
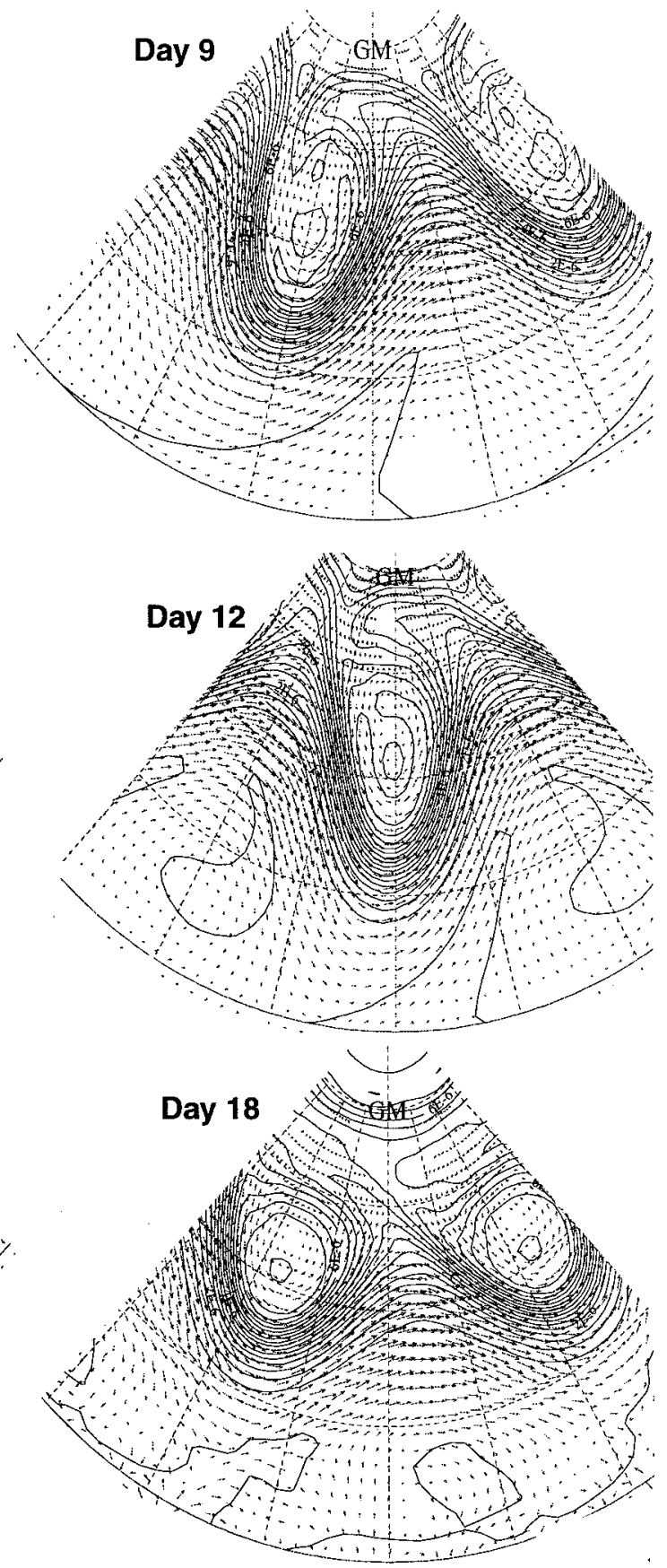

FIG. 6. As in Fig. 4 except for the case of $u_{0}=8.25 \mathrm{~m} \mathrm{~s}^{-1}$.

will not go where the index of refraction goes to zero, which occurs for $K \sim m$. We have estimated the phase speed from the eastward movement of the PV minima along latitude circles from $38^{\circ}$ to $43^{\circ}$ during the period of linear growth, rather than using the normal mode phase speed as THM did. The phase speed changes approximately linearly from 10.5 to $5.7 \mathrm{~m} \mathrm{~s}^{-1}$ as the shear parameter is increased from 0 to $10 \mathrm{~m} \mathrm{~s}^{-1}$. Changes in wind speed and resulting changes in phase speed are both important in determining the response of the index 

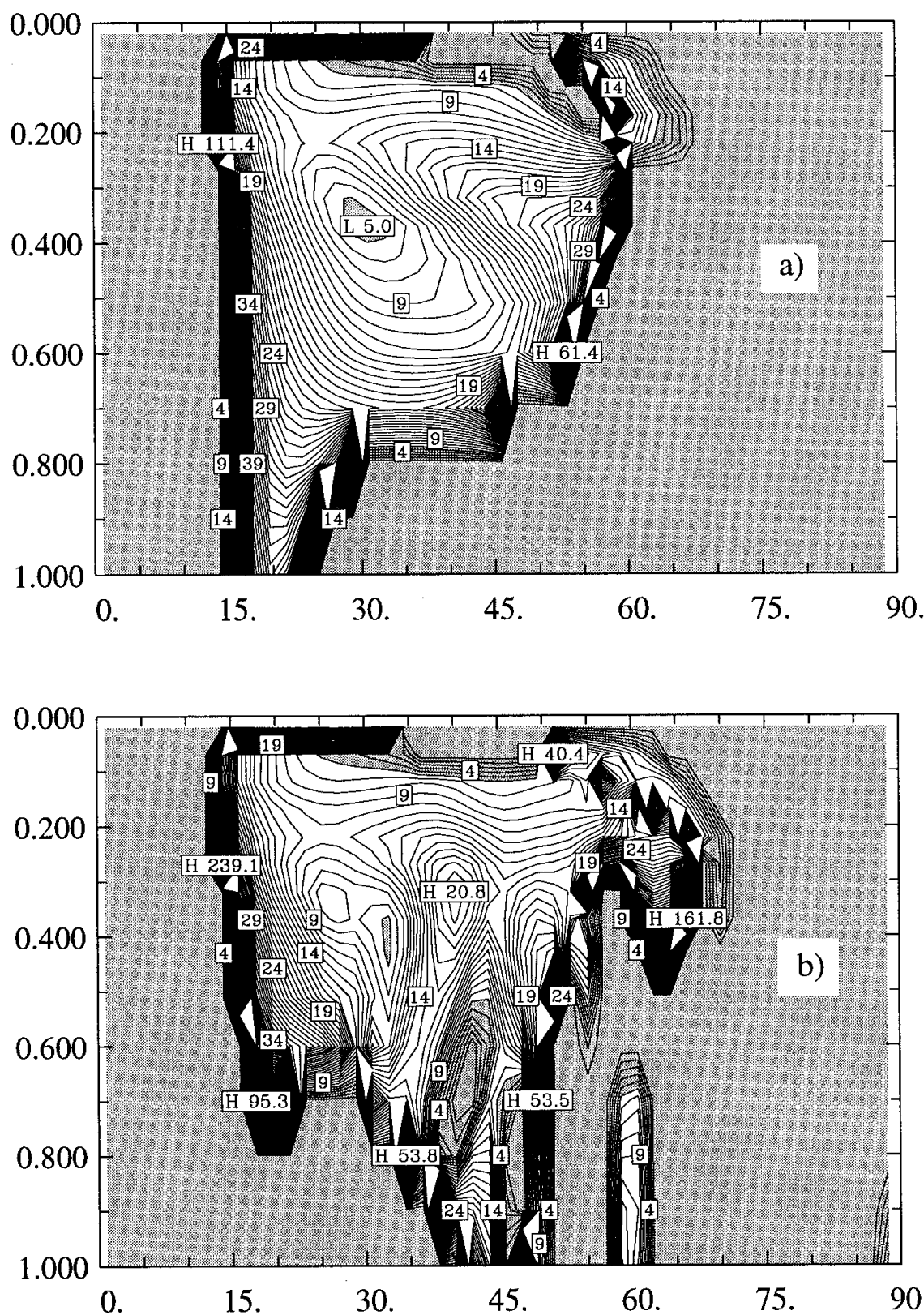

FIG. 7. Modified index of refraction cross section for the case of $u_{0}=7.5 \mathrm{~m} \mathrm{~s}^{-1}$ at (a) day 0 and (b) day 7. Contour interval is 1 and values less than 6 are shaded.

of refraction to barotropic shear. As THM showed, the changes in $\bar{q}_{y}$ are of tertiary importance.

In Figs. 7 and 8 we show the modified index of refraction, $K$, for the cases $u_{0}=7.5$ and $8.25 \mathrm{~m} \mathrm{~s}^{-1}$, respectively, at day 0 and day 7 . Day 7 is near the end of the period of normal mode growth, and about the last day for which we expect linear theory to be useful. THM noted two significant differences between their anticyclonic $\left(u_{0}=0.0\right)$ and cyclonic $\left(u_{0}=10.0\right)$ cases. In the cyclonic case, the critical line is moved much deeper into the Tropics, and the minimum in $K$ on the equatorward flank of the jet gets smaller than 6 , so that wavenumber 6 should be evanescent in that region, according to linear theory. We also see these differences very clearly when we compare the $u_{0}=0.0$ case (not shown) with either the $u_{0}=7.5$ or the $u_{0}=8.25$ case. The index of refraction does not suggest the abruptness of the transition from anticyclonic to cyclonic behavior in the model, however, since it is difficult to see much that is significantly different between the modified index of refraction plots in Figs. 7 and 8, though one exhibits clearly anticyclonic and the other clearly cyclonic behavior.

An important feature of the two cases shown is that 

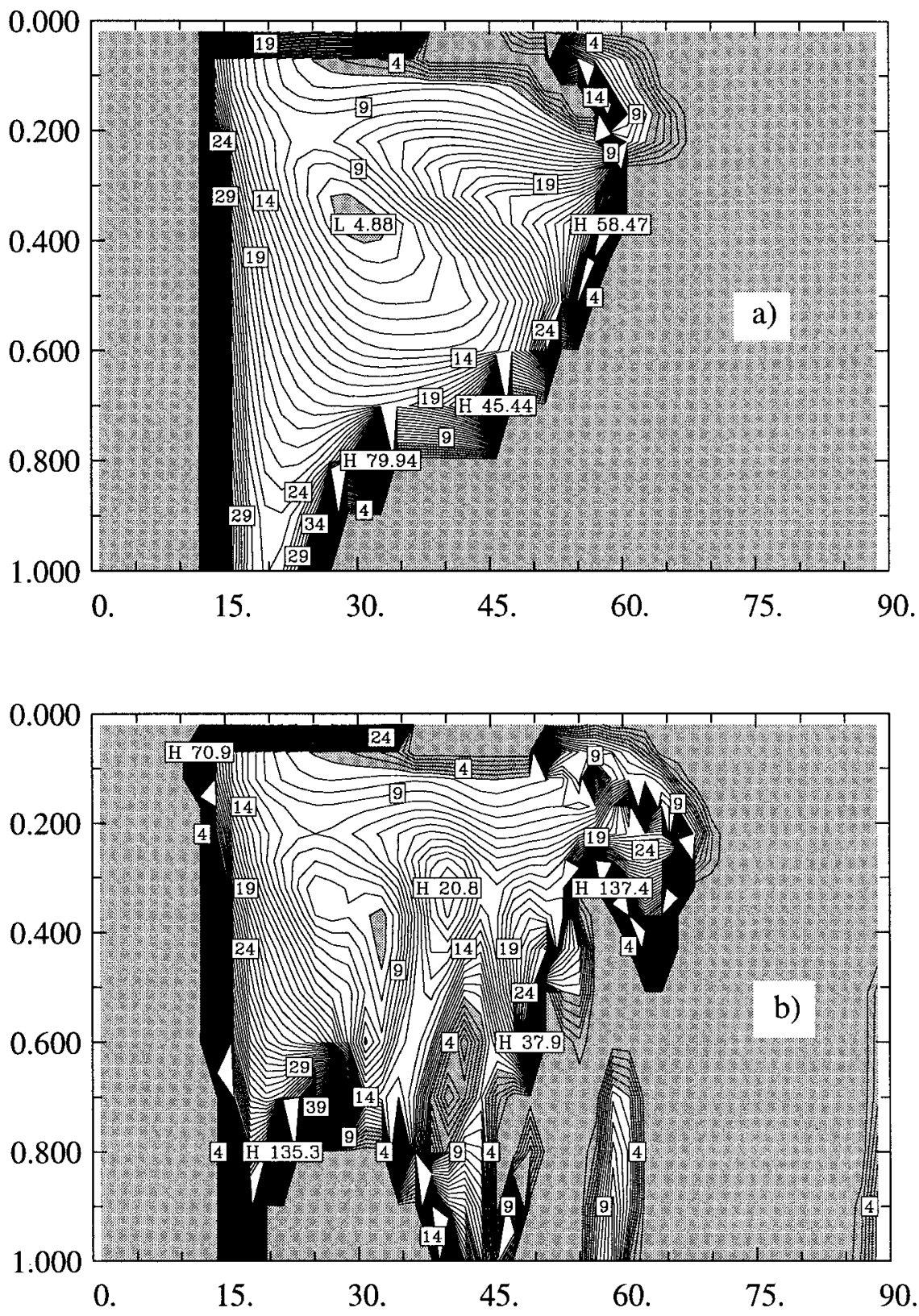

FIG. 8. As in Fig. 7 except for case $u_{0}=8.25 \mathrm{~m} \mathrm{~s}^{-1}$.

as the flow evolves the minimum at about $30^{\circ}$ is maintained and extended vertically, so that by day 7 a very strong gradient in $K$ occurs between values less than 6 at about $30^{\circ}$ and values greater than 20 at about $40^{\circ}$. This gradient extends vertically from the base of the propagating region to about $300 \mathrm{mb}$. WKB theory predicts that such a strong gradient will refract waves poleward as they propagate upward. This gradient, combined with the fact that the critical line in the upper troposphere has been moved from about $30^{\circ}$ to about $15^{\circ}$ by the addition of significant barotropic cyclonic shear, may help to explain why much less equatorward eddy propagation and wave breaking occurs as the barotropic shear parameter is increased.

It is interesting to plot the magnitude of the $K$ minimum on the equatorward flank of the jet at day 0 and day 7 as functions of the shear parameter (Fig. 9). For low values of the shear parameter, the $K$ minimum is washed out by the wave-mean flow interactions during the first 7 days. As the shear parameter is increased, the $K$ minimum gets smaller at initial time and the wavemean flow interactions also tend to sustain this minimum better so that $K$ stays below the interesting value of 6 for all of the first 7 days. This is evidence of positive 


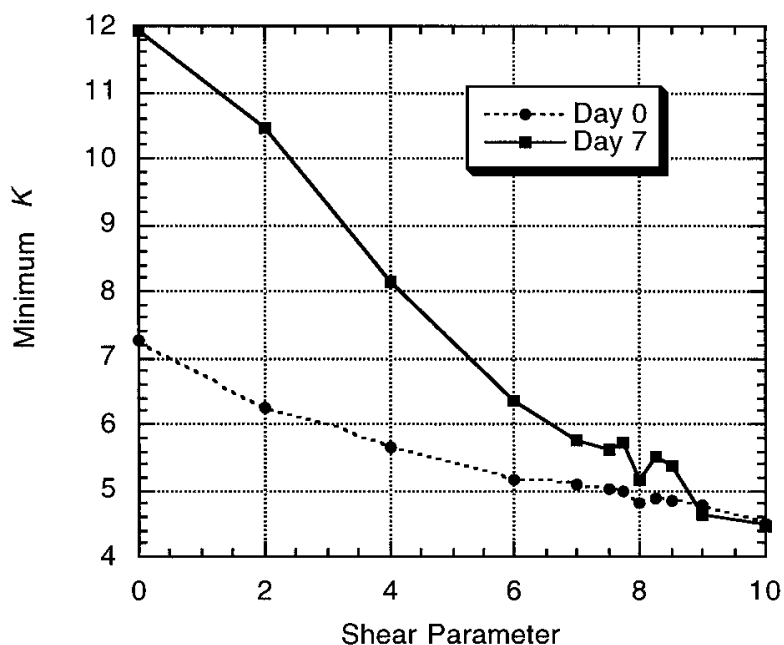

FIG. 9. Values of the internal minimum in $K$ at about $30^{\circ}$ lat and $400 \mathrm{mb}$ as a function of the shear parameter $u_{0}$ at day 0 and day 7 .

feedback between the impedance of equatorward propagation and mean-flow accelerations early in the life cycle. The minimum $K$ value at day 7 provides a suggestive, but not very precise, prediction of the transition from anticyclonic to cyclonic behavior. The minimum value stays below the critical value of 6 for all shear parameters greater than about 6.5 , but the transition taken place abruptly between shear parameters of 7.5 and 8.25.

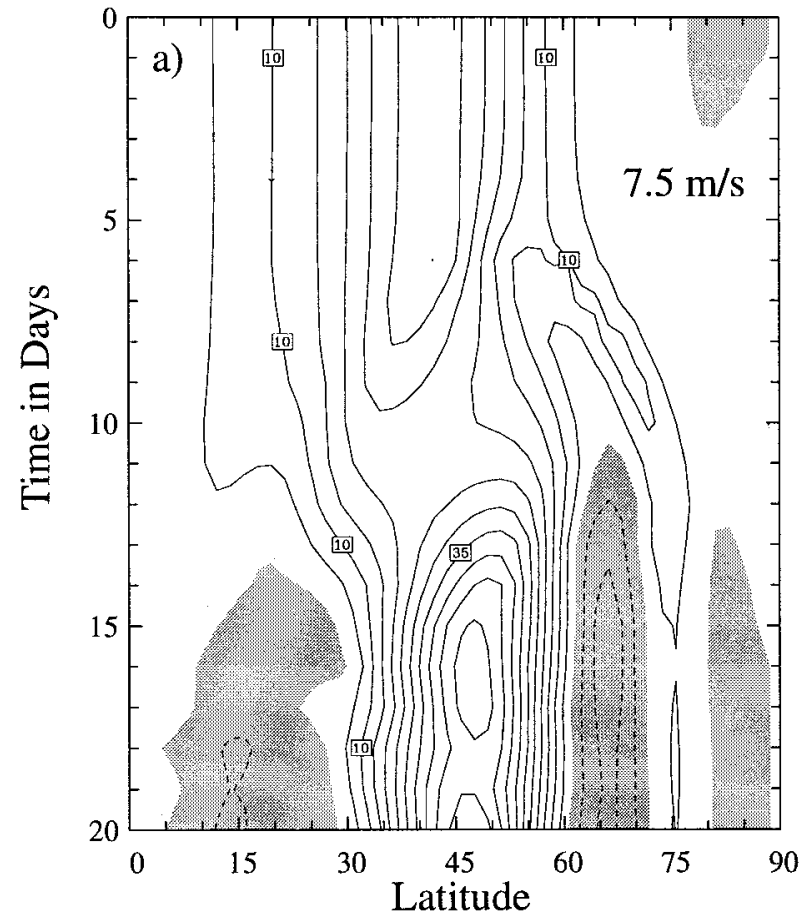

\section{b. Zonal wind accelerations}

It is interesting to compare the evolution of the zonally averaged wind in the upper troposphere in the two cases of most interest. Figure 10 shows the temporal evolution of the wind near $300 \mathrm{mb}$ for the $u_{0}=7.5$ and $u_{0}=8.25$ cases. Between day 7 and day 15, in the anticyclonic $u_{0}=7.5$ case the westerly jet first weakens and then shifts poleward by about $5^{\circ}$ and intensifies. For the cyclonic $u_{0}=8.25$ case, the jet weakens less and shifts slightly equatorward. The jet core moves rapidly near the time of wave saturation, and between days 11 and 13 the jet core moves to the poleward flank of the EKE maximum in the anticyclonic case but stays equatorward of the EKE maximum in the cyclonic case (not shown).

The total zonal flow accelerations can be diagnosed using the transformed Eulerian mean (TEM) equations:

$$
\frac{\partial \bar{u}}{\partial t} \approx f \bar{v}^{*}+\frac{1}{a \cos \varphi} \nabla \cdot \mathbf{F}+\text { friction }+\cdots,
$$

where $\bar{v}^{*}$ is the residual mean meridional circulation and $\mathbf{F}$ is the Eliassen-Palm (EP) flux vector (Edmon et al. 1980). The first two terms on the right-hand side of (6) describe the Coriolis acceleration by the residual circulation and the eddy driving by the EP flux divergence. For strongly transient cases such as a baroclinic life cycle, a large degree of cancellation exists between the EP flux divergence and the Coriolis acceleration by the residual circulation, so it is most meaningful to plot

FIG. 10. Latitude vs time contour plot of the zonal mean wind on the $\sigma=0.32$ surface for the (a) $u_{0}=7.5 \mathrm{~m} \mathrm{~s}^{-1}$ and (b) $u_{0}=8.25 \mathrm{~m}$ $\mathrm{s}^{-1}$ cases. The contour interval is $5 \mathrm{~m} \mathrm{~s}^{-1}$ and negative zonal winds are shaded. 

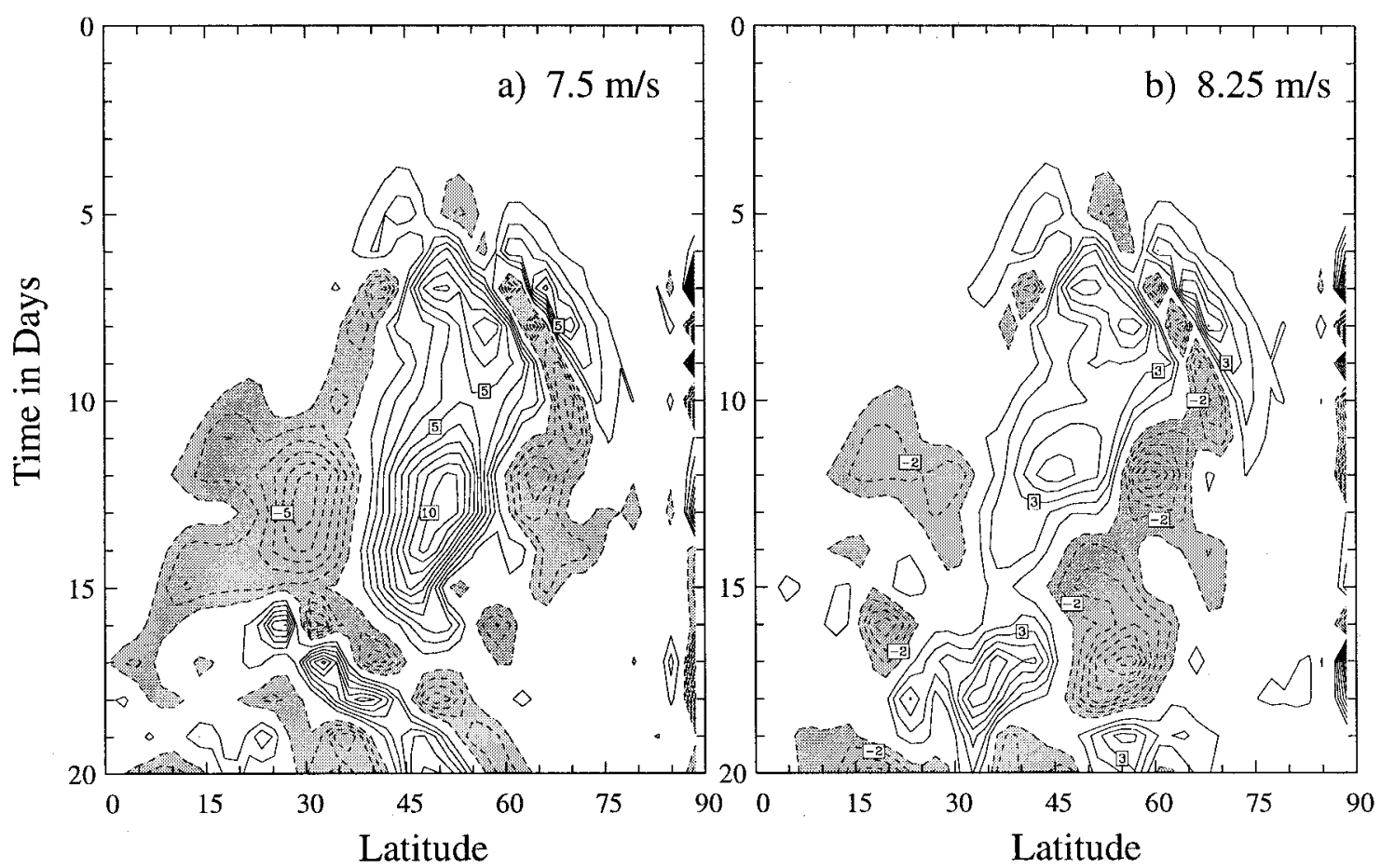

FIG. 11. As in Fig. 10 except the quantity plotted is the total advective forcing of zonal wind at $\sigma=0.32$. The contour interval is $1 \mathrm{~m}$ $\mathrm{s}^{-1}$ day $^{-1}$ and values less than $-1 \mathrm{~m} \mathrm{~s}^{-1}$ day $^{-1}$ are shaded. The zero contour is omitted.

the sum of the two terms, which we will call the total advective forcing. Also, as Yu and Hartmann (1993) noted, while the EP flux divergences show strong signals in the upper troposphere associated with meridional eddy fluxes, the forcing of zonal flow by the residual circulation partially cancels the effect of EP flux divergence, and the total advective forcing can be much weaker and of a very different shape than the EP flux divergence alone.

In Figure 11 we show the total advective forcing of the zonal mean flow at the $\sigma=0.32$ level as a function of latitude and time for the two cases of $u_{0}=7.5$ and $8.25 \mathrm{~m} \mathrm{~s}^{-1}$, the anticyclonic and cyclonic cases, respectively. The forcing is very similar up to about day 11 , after which a strong forcing dipole occurs between days 12 and 15 in the anticyclonic case but not in the cyclonic case. An examination of the components of the advective forcing shows that this dipole structure derives mostly from the convergence of the meridional transport of momentum by the eddy. This forcing dipole has centers at about $30^{\circ}$ and $50^{\circ}$ of latitude and strongly forces anticyclonic shear in this latitude belt in the anticyclonic case. In the cyclonic case the westerly acceleration is weaker and closer to the equator, and tends to sustain or enhance the original cyclonic shear. A dipole of cyclonic forcing appears in the cyclonic case around day 17.

Cross sections of the EP flux vectors and their divergence (Edmon et al. 1980) show very clear signatures associated with the transition from anticyclonic to cy- clonic behavior (THM) as do wave activity advection diagnostics (Magnusdottir and Haynes 1996). We show here a key element of the transition between the $u_{0}=$ 7.5 and $u_{0}=8.25 \mathrm{~m} \mathrm{~s}^{-1}$ cases using the EP cross section at day 13 (Fig. 12). The EP flux vectors and their divergences are very similar for both cases up to about day 10, but then develop very dramatic differences. At day 13 in the $u_{0}=7.5$ case the EP flux vectors point equatorward in the upper troposphere and with this signature are associated wave breaking and a dipole pattern in the eddy flux driving of mean-flow acceleration. The nature of this acceleration is to drive westerlies around $50^{\circ}$ and easterlies around $30^{\circ}$, and when combined with the Coriolis acceleration it produces the net advective forcing differences described below. In the case of $u_{0}$ $=8.25$ very little meridional component to the EP flux vectors develops and the eddy acceleration of zonal flow is much weaker. The eddy activity appears to be trapped in a cavity from which there is little leakage. So the transition from equatorward propagation and wave breaking to trapped, wave cavity types of behaviors occurs for a rather small shift in the barotropic shear parameter.

To show the vertical structure of the accelerations, the cross sections of total advective forcing averaged over days $1-11$ and over days $12-15$ are shown in Fig. 13. The driving of zonal flow accelerations over the first 11 days shows small differences between the two cases. The total advective forcing during the critical day 1215 period is very different in the two cases. In the an- 


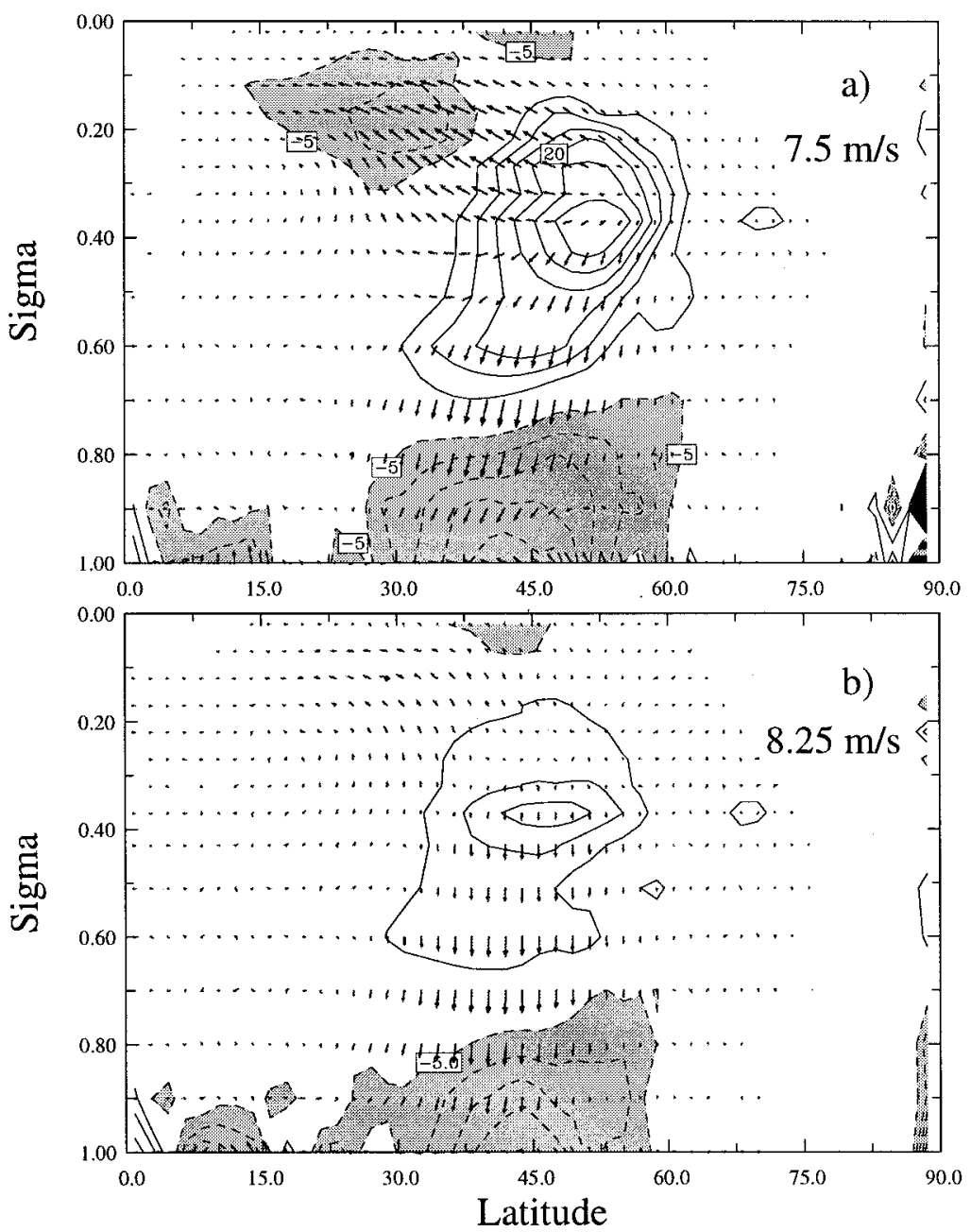

FIG. 12. Zonal cross sections of EP flux vectors and eddy zonal flow acceleration at day 13 in the (a) $u_{0}=7.5 \mathrm{~m} \mathrm{~s}^{-1}$ and (b) $u_{0}=8.25 \mathrm{~m} \mathrm{~s}^{-1}$ cases. The contour interval is $5 \mathrm{~m} \mathrm{~s}^{-1}$ day $^{-1}$ and values less than -5 are shaded. The vector scale is the same in both panels.

ticyclonic case a strong anticyclonic dipole of forcing exists with westerlies driven at $50^{\circ}$ lat and easterlies at $25^{\circ}$ lat, but in the cyclonic case weak westerly accelerations are driven at $40^{\circ}$ lat. The accelerations near the lower boundary in the Tropics are associated with secondary baroclinic instability and are not important for the midlatitude wave life cycle.

To summarize the differences in zonal flow acceleration over the whole life cycle, Fig. 14 shows a zonal cross section of the change in zonal mean flow between day zero and day 20 for $u_{0}=7.5$ and $8.25 \mathrm{~m} \mathrm{~s}^{-1}$. In both cases the change in zonal flow has a half-wavelength of about $20^{\circ}$ of lat and rather weak variations of phase and amplitude with height. In the anticyclonic case with the weaker initial barotropic shear, the westerly acceleration is stronger and occurs at a higher latitude, centered at about $48^{\circ}$, whereas in the cyclonic case it is centered at about $40^{\circ}$. It is a measure of the abruptness of the transition from anticyclonic to cy- clonic flow that the difference in the net acceleration between the cases for shear parameters of 7.5 and 8.25, is much larger than the difference between the net acceleration for the 7.5 and 0.0 cases (not shown).

\section{Summary and discussion}

A cyclonic barotropic wind perturbation of gradually increasing magnitude is added to a baroclinic midlatitude jet to create the initial conditions for a series of 20-day baroclinic life cycle experiments. While some of the properties of the simulations show a linear response to added barotropic shear, the life cycle behavior shifts from anticyclonic to cyclonic across a very narrow threshold of barotropic shear. The sharpness of the transition can be seen in several important characteristics of flow: the fraction of the maximum eddy kinetic energy that is retained at the end of the integration, the barotropic energy conversion integrated over the 20 

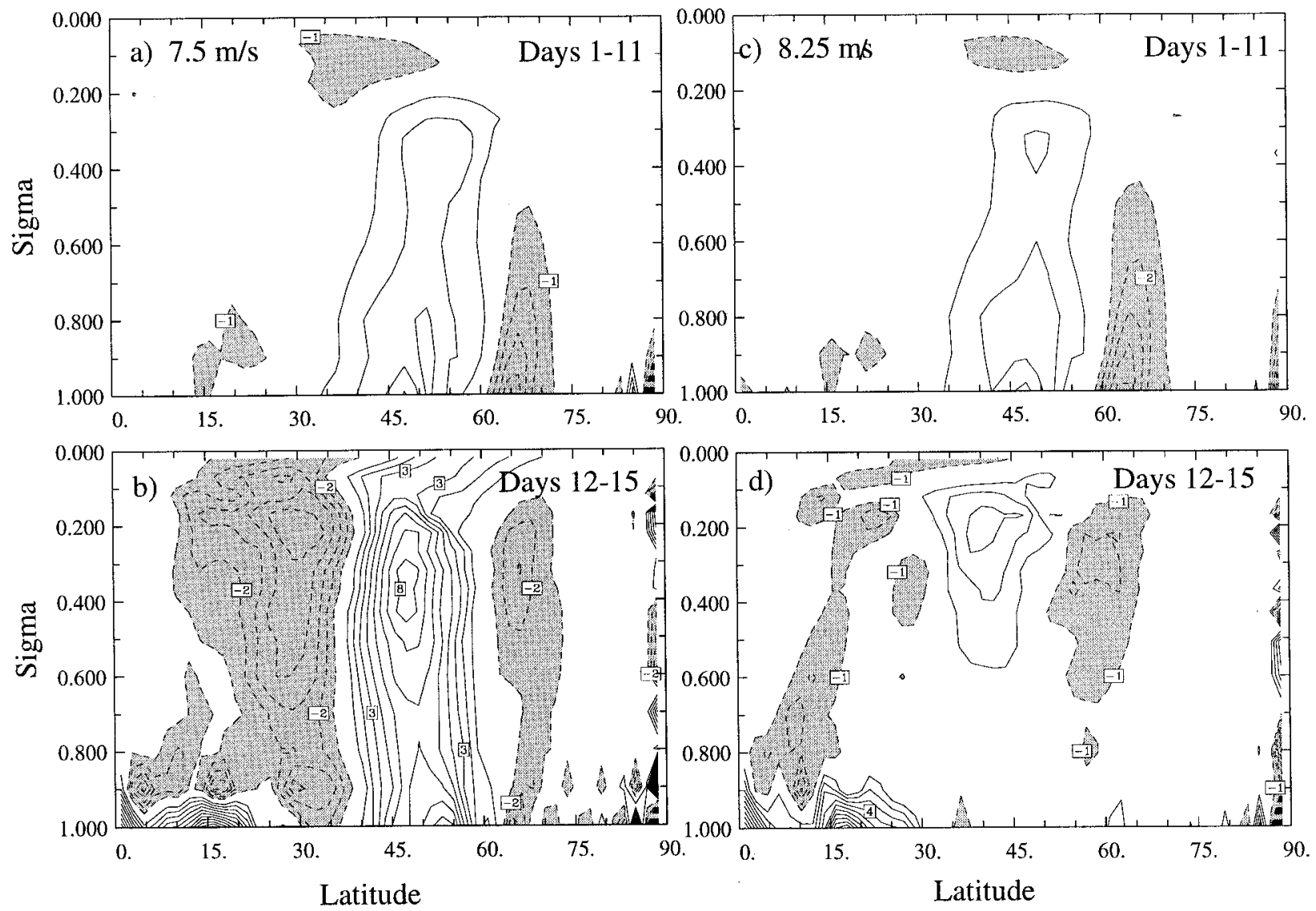

FIG. 13. Zonal cross sections of total advective forcing for the $u_{0}=7.5 \mathrm{~m} \mathrm{~s}^{-1}(\mathrm{a}, \mathrm{b})$ and $u_{0}=8.25 \mathrm{~m} \mathrm{~s}^{-1}$ (c, d) cases averaged over days $1-11$ (a, c) and days 12-15 (b, d). Contour interval is $1 \mathrm{~m} \mathrm{~s}^{-1}$ day $^{-1}$, the zero contour is omitted, and values less than $-1 \mathrm{~m} \mathrm{~s}^{-1}$ day ${ }^{-1}$ are shaded.

days of the experiment, the change in the zonal flow over the life cycle, and the evolution of the potential vorticity field.

It is perhaps helpful to think of the sharpness of the transition from anticyclonic to cyclonic behavior as a result of the strong feedback between eddy propagation and zonal flow. Below the threshold, zonal flow accelerations reduce the initial barotropic cyclonic curvature and thus push the flow evolution toward the anticyclonic behavior observed for initial conditions with smaller barotropic shears. The zonal flow accelerations are particularly dramatic in the final stages of the life cycle, but linear theory suggests that some subtle, but important, changes may occur during the growth phase of the wave as well. Once the initial cyclonic shear crosses a threshold value, the wave cannot easily reach a critical surface on the equatorward flank of the jet, much less wave breaking occurs on the equatorward flank of the jet, and the resulting zonal flow accelerations reinforce the initial barotropic cyclonic shear.

Although the conditions for the WKB approximation are not strictly valid for the conditions of these numerical experiments, linear wave propagation theory, as encapsulated in the index of refraction for Rossby waves, appears to give useful predictions of the response of the propagation characteristics to barotropic shear, at least in the early part of the life cycle, as THM suggested. The added barotropic shear, and the changes in wave phase speed that accompany it, produce changes in distribution of the index of refraction in latitude-height cross sections that suggest why the eddy is less likely to find critical lines on the equatorward flank of the jet when barotropic shear is added. Increasing barotropic shear gives rise to a region of low index of refraction at about $30^{\circ}$ lat and the critical surface for wavenumber 6 is moved well equatorward of this minimum. This suggests that waves will be refracted back poleward before they reach a critical surface where wave breaking is likely. Index of refraction calculations also hint at the nature of the positive feedback process since zonal flow accelerations during the growth phase of the wave seem to reduce the impedance to equatorward wave propagation when the initial barotropic shear is weak, but the accelerations seem to increase the impedance of equatorward propagation when the initial barotropic shear is stronger. Linear wave theory does not provide a very accurate prediction of either the existence of a sharp transition or the shear parameter at which it occurs, however.

Index of refraction calculations also suggest that the 

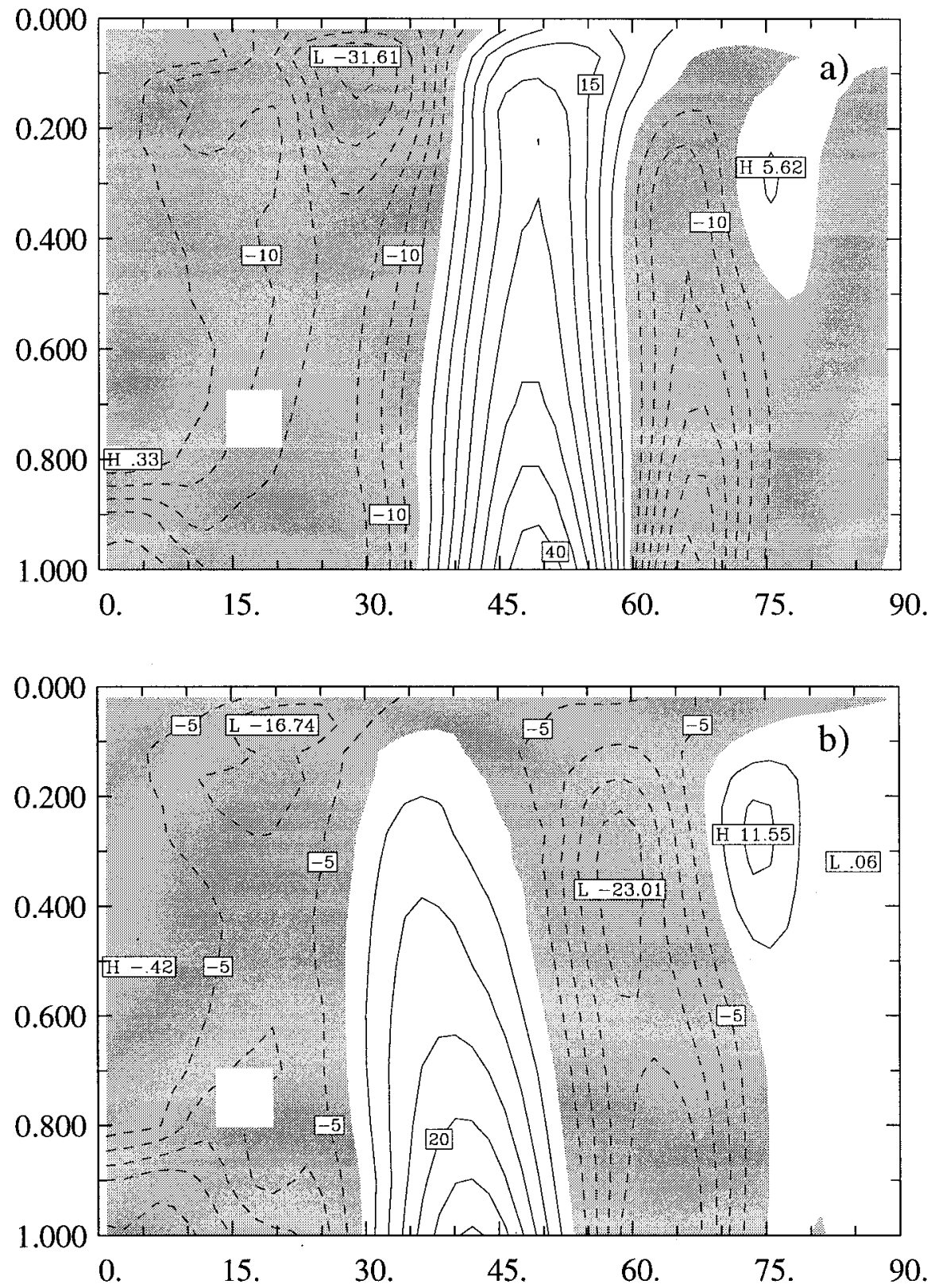

Fig. 14. Zonal cross sections of the change in zonal mean wind between day 0 and day 20 for the (a) $u_{0}=7.5 \mathrm{~m} \mathrm{~s}^{-1}$ and (b) $u_{0}=8.25 \mathrm{~m} \mathrm{~s}^{-1}$ cases. Contour interval is $5 \mathrm{~m} \mathrm{~s}^{-1}$ and negative values are shaded.

effect of barotropic shear and the threshold for transition to cyclonic behavior will depend sensitively on the zonal wavenumber chosen for the experiment. If a higher wavenumber is chosen, equatorward propagation will be inhibited for even smaller shears. If a smaller wavenumber is chosen, then equatorward propagation will be possible even for large barotropic shear. We have conducted a few experiments to illustrate that these inferences are correct. We did a series of experiments for wavenumbers 4 and 8 . The evolution of the eddy kinetic energy for these wavenumbers with shear parameters of
0 and 10 are shown in Fig. 15 along with the results shown previously for wavenumber 6 . Wavenumber 8 takes longer to grow and reaches a lower value of eddy kinetic energy, but its eddy kinetic energy maintains a substantial fraction of its peak value all the way to day 20 and beyond. Its behavior is cyclonic for both strong and weak barotropic shear. Wavenumber 4 takes a long time to grow. It reaches a high kinetic energy, but its energy tends to decrease rapidly after it reaches its peak. Potential vorticity maps indicate that wavenumber 4 experiences significant wave breaking on the equatorward 


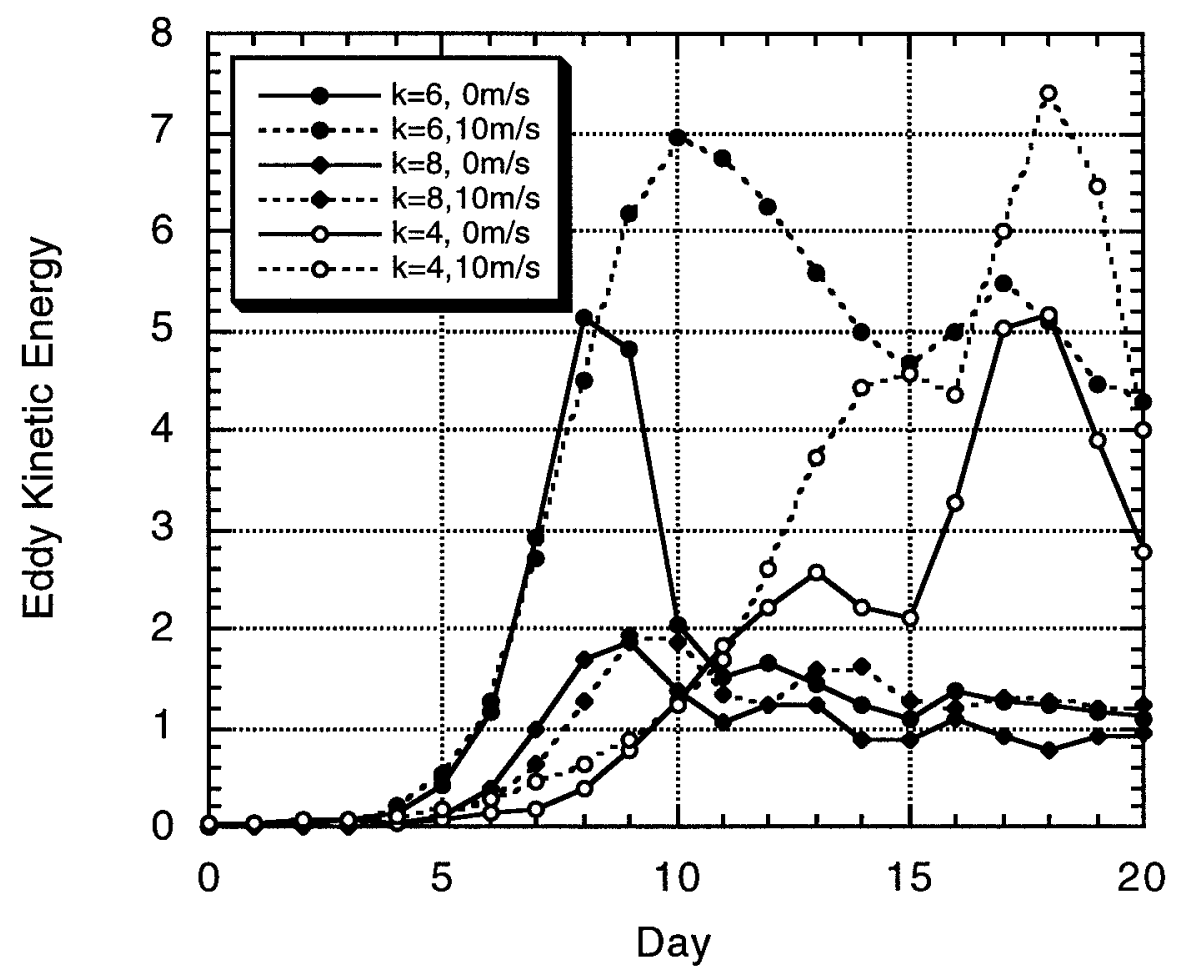

FIG. 15. Integrated eddy kinetic energy as a function of time for experiments with $u_{0}=0.0$ or 10 $\mathrm{m} \mathrm{s}^{-1}$ and zonal wavenumbers 4,6 , and 8 .

flank of the jet for all values of cyclonic shear. Its behavior is anticyclonic for both low and high barotropic shear.

One might reasonably argue that the strong sensitivity of the behavior to zonal wavenumber will make the transition between anticyclonic and cyclonic behavior less important in real-world situations than in these idealized experiments, since a flow that is at a transition point for one zonal scale may be very far from the transition point for another. On the other hand, Fig. 15 suggests that the wavenumber that shows the strongest transition sensitivity is also the one that achieves the highest amplitude, probably for reasons closely related to those for the transition itself. The wave grows rapidly, propagates easily up to the tropopause, and is near the margin where equatorward wave propagation and breaking is partially inhibited. Therefore, we are tempted to speculate that the wavenumbers that are most energetic for a particular zonal flow configuration are also the wavenumbers that are most likely to be near their transition from cyclonic to anticyclonic behavior for that same flow. We are currently attempting to test this speculation using realistic Southern Hemisphere flows.

We are interested in the sharpness of the transition from anticyclonic to cyclonic behavior because it provides a mechanism through which synoptic-scale eddies and zonal flow can interact with each other to produce low-frequency variability of zonal flow, as has been proposed by Yu and Hartmann (1993) and Hartmann
(1995). Eddies growing on a zonal flow with a barotropic shear below the critical value will tend to develop weaker barotropic shears and therefore move away from the transition point, whereas those finding themselves in stronger barotropic shears above the threshold will act to enhance the barotropic shear. This provides a mechanism whereby a wide range of barotropic shears can be equally viable quasi-steady flow states. Since the distribution of surface winds with latitude varies significantly between the hypothesized quasi-equilibrium states, this provides a potentially interesting interaction with the ocean on long timescales.

The sharpness of the transition also has interesting implications for medium and extended range forecasting since it may make forecasts a week or more into the future very sensitive to small changes in the initial or forecast barotropic shear. The strong feedback between the horizontal tilt of the eddies and the mean barotropic flow also makes the sources of systematic errors in jet stream winds difficult to diagnose.

Acknowledgments. This work was supported by the Climate Dynamics Program, National Science Foundation under Grant 93-13383. We thank Marc Michelsen, Candace Gudmundson, and Melissa Rogers for their assistance. The anonymous reviewers provided many useful comments that improved the paper. 


\section{REFERENCES}

Edmon, H. J., Jr., B. J. Hoskins, and M. E. McIntyre, 1980: EliassenPalm cross sections for the troposphere. J. Atmos. Sci., 37, 26002616; Corrigendum, 38, 1115.

Hartmann, D. L., 1995: A PV view of zonal flow vacillation. J. Atmos. Sci., 52, 2561-2576.

Haynes, P. H., 1988: Forced, dissipative generalizations of finiteamplitude wave-activity conservation relations for zonal and nonzonal basic flows. J. Atmos. Sci., 45, 2352-2362.

Hoskins, B. J., and D. J. Karoly, 1981: The steady-state linear response of a spherical atmosphere to thermal and orographic forcing. J. Atmos. Sci., 38, 1179-1196.

- M. E. McIntyre, and A. W. Robertson, 1985: On the use and significance of isentropic potential-vorticity maps. Quart. J. Roy. Meteor. Soc., 111, 877-946.

James, I. N., 1987: Suppression of baroclinic instability in horizontally sheared flows. J. Atmos. Sci., 44, 3710-3720.

—, and J. P. Dodd, 1996: A mechanism for the low-frequency variability of the mid-latitude troposphere. Quart. J. Roy. Me teor. Soc., 122, 1197-1210.

Karoly, D., and B. J. Hoskins, 1982: Three-dimensional propagation of planetary waves. J. Meteor. Soc. Japan, 60, 109-123.
Lorenz, E. N., 1964: The problem of deducing the climate from the governing equations. Tellus, 16, 21-31.

- 1967: The Nature and Theory of the General Circulation of the Atmosphere. World Meteorological Organization, 161 pp.

Magnusdottir, G., and P. H. Haynes, 1996: Wave activity diagnostics applied to baroclinic wave life cycles. J. Atmos. Sci., 53, 23172353.

Matsuno, T., 1970: Vertical propagation of stationary planetary waves in the winter Northern Hemisphere. J. Atmos. Sci., 27, 871-883.

Simmons, A. J., and B. J. Hoskins, 1980: Barotropic influences on the growth and decay of nonlinear baroclinic waves. J. Atmos. Sci., 37, 1679-1684.

Thorncroft, C. D., B. J. Hoskins, and M. E. McIntyre, 1993: Two paradigms of baroclinic life-cycle behaviour. Quart. J. Roy. Meteor. Soc., 119, 17-55.

Yu, J.-Y., and D. L. Hartmann, 1993: Zonal flow vacillation and eddy forcing in a simple GCM of the atmosphere. J. Atmos. Sci., 50, 3244-3259.

Zuercher, P., 1996: Bimodal response of baroclinic wave lifecycles to changes in horizontal, barotropic shear. M.S. thesis, Dept. of Atmospheric Sciences, University of Washington, $114 \mathrm{pp}$. [Available from Resource Sharing, Suzzallo and Allen Library, Box 352900, University of Washington, Seattle, WA 92195.] 